\title{
A Fuzzy Logic Solar Controller with Maximum Power Point Tracking
}

\author{
Oleg A. Dontsov*, \\ Vladimir I. Ivanchura and Yuri V. Krasnobaev \\ Siberian Federal University \\ 79 Svobodny, Krasnoyarsk, 660041, Russia
}

Received 23.05.2015, received in revised form 06.09.2015, accepted 09.09.2015

This article presents the discussion and comparison of two solar controller operation algorithms. According to these algorithms, the solar controller can operate in maximum power point tracking mode and battery charging mode, which is characterized by the maintaining an optimal charging current with the limiting of battery voltage. The paper presents the block diagrams of the maximum power point tracking mode algorithm and battery charging algorithm. The study of processes in an autonomous power supply system using the physical layout and simulation model, showed the correctness of the proposed algorithms. A quantitative comparison of the efficiency of the power supply system was made using the constant and variable step maximum power point tracking algorithms.

Keywords: solar cell, solar controller, battery, maximum power point tracking, autonomous power supply system, fuzzy logic.

DOI: $10.17516 / 1999-494 X-2015-8-6-786-794$.

\section{Контроллер солнечной батареи с экстремальным регулированием на основе нечеткой логики}

\author{
О.А. Донцов, В.И. Иванчура, Ю.В. Краснобаев \\ Сибирский федеральный университет \\ Россия, 660041, Красноярск, пр. Свободньій, 79
}

\begin{abstract}
В статье рассматриваются два алгоритма функиионирования контроллера солнечной батареи автономной системы электропитания и проводится их сравнительная оценка. В соответствии с алгоритмами контроллер солнечной батареи может работать как в режиме поиска экстремальной мощности солнечной батареи, так и в режиме заряда аккумуляторной батареи заданным током при ограничении зарядного напряжения. Приведены блок-схемы разработанных алгоритмов заряда аккумуляторной батареи и поиска экстремальной мощности солнечной батареи. Проведено исследование процессов 6
\end{abstract}

(C) Siberian Federal University. All rights reserved
* Corresponding author E-mail address: badzed_bz@mail.ru 
автономной системе электропитания с использованием физического макета и имитационной модели, показавщее правильность предложенных алгоритмов. Выполнено количественное сравнение эффективности работы системы электропитания при использовании алгоритмов поиска экстремальной мощности с постоянным и переменным шагом.

Ключевые слова: солнечная батарея, контроллер солнечной батареи, аккумуляторная батарея, экстремальное регулирование мощности, автономная система электропитания, нечеткая логика.

\section{1. Введение}

Автономные системы электропитания (СЭП) на основе солнечных батарей (СБ) находят все большее применение как в районах, удаленных от централизованной сети энергоснабжения, так и в ряде стран, имеющих высокий уровень солнечной радиации и развивающих альтернативные источники энергоснабжения для снижения зависимости от поставок энергоносителей. В настоящее время существует значительное количество международных организаций и проектов, целью которых является содействие широкому внедрению и использованию возобновляемых источников энергии, а также распространение информации, знаний и технологий в этой области [1]. Постоянное снижение стоимости СБ, происходящее последние несколько лет $[2,3]$, также приводит к более интенсивному использованию солнечной энергии в СЭП [4].

\section{2. Постановка задачи}

Системы электропитания постоянного тока на основе солнечной батареи включают в себя непосредственно СБ, аккумуляторную батарею (АБ), кабельную сеть и контроллер СБ, выполненный в виде конвертора с устройством управления. До недавнего времени функции, выполняемые контроллером СБ в наземных автономных СЭП, сводились к обеспечению регулирования потока энергии от СБ в АБ и нагрузку из условий ограничения величины зарядного тока АБ на требуемом уровне и дозаряда АБ снижающимся током при некотором заданном максимальном напряжении на АБ. Только в СЭП космических аппаратов в случае, когда сумма мощности, потребляемой нагрузкой, и мощности, идущей на заряд АБ, превышала текущую мощность, генерируемую СБ, контроллер СБ переводился в режим экстремального регулирования, позволяющий получать от СБ максимально возможную мощность [5]. В последнее время стали выпускаться контроллеры для СЭП широкого применения с функцией отбора экстремальной мощности, которые дают возможность увеличить мощность, генерируемую СБ, на $15-30 \%$ [5].

В режиме экстремального регулирования рабочая точка на мощностной характеристике СБ совершает колебания в окрестности точки максимальной мощности. При этом часть мощности СБ, превышающая мощность, потребляемую нагрузкой, поступает на заряд АБ. Современное развитие микроконтроллерной техники позволяет реализовать управление контроллером СБ на основе микроконтроллера (МК). При этом появляется возможность реализовать не только пошаговый алгоритм поиска экстремума мощности СБ [5-7], но и более сложные алгоритмы, помогающие ускорить процесс поиска экстремума и повысить уровень мощности, генерируемой СБ за счет снижения амплитуды колебаний рабочей точки на вольтамперной характеристике СБ в окрестности точки максимальной мощности. Один из таких алгоритмов и 
рассматривается в настоящей статье, проводится его сравнительная оценка с пошаговым алгоритмом поиска экстремума мощности СБ.

\section{3. Решение задачи}

Блок-схема алгоритма функционирования контроллера СБ, который обеспечивает реализацию режимов заряда АБ оптимальным током, приведена на рис. 1. Значения оптимального тока заряда, а также минимального и максимального напряжения на выводах АБ задаются программно в соответствии с типом и емкостью аккумуляторной батареи.

Согласно алгоритму контроллер считывает сигналы напряжения и тока АБ, после чего выполняется проверка равенства текущего значения тока и заданного значения оптимального тока заряда. Если текущее значение тока находится в некотором заданном интервале, т.е. равно оптимальному зарядному току с некоторой погрешностью, коэффициент заполнения импульсов управления силового ключа конвертора остается неизменным. Если ток заряда меньше заданного с учетом погрешности, то происходит увеличение коэффициента заполнения импульсов управления, а в противном случае он становится меньше. Далее происходит проверка условия достижения напряжения на АБ своего максимального значения. Если это условие соблюдается, то идет уменьшение коэффициента заполнения импульсов управления пропорционально дальнейшему увеличению напряжения на АБ.

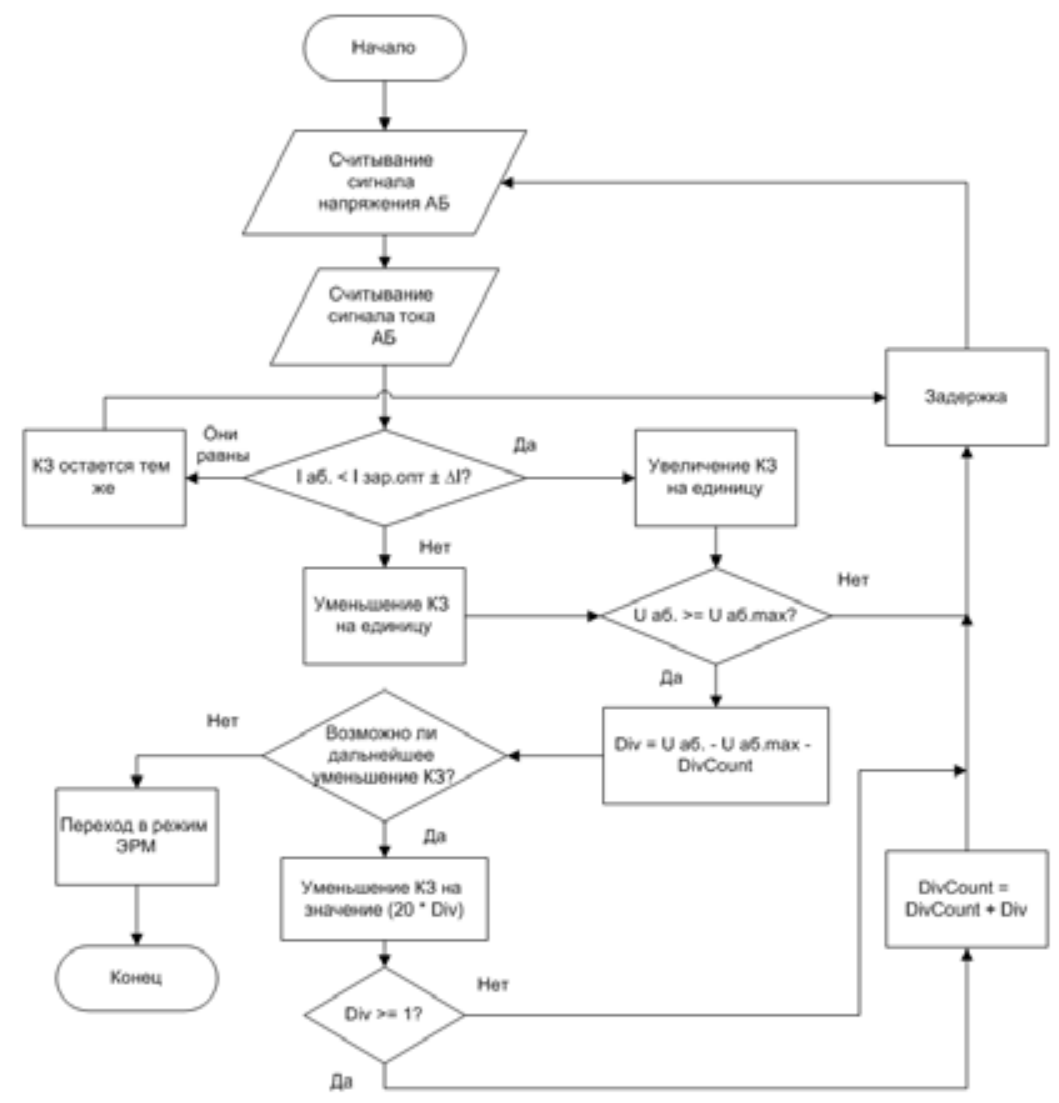

Рис. 1. Алгоритм работы режима заряда АБ оптимальным током 


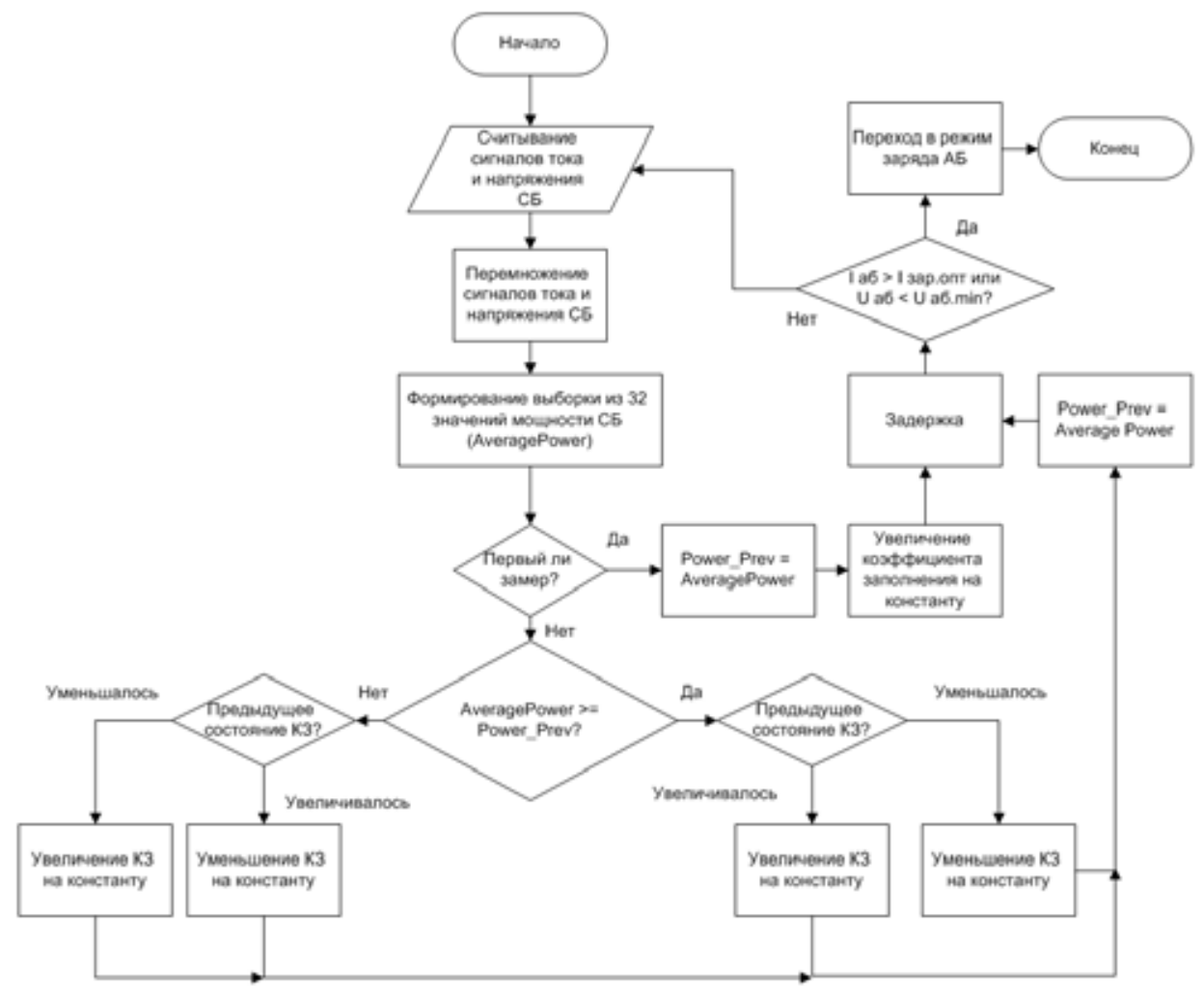

Рис. 2. Алгоритм работы режима поиска экстремальной мощности с фиксированным шагом

На рис. 2 приведена блок-схема пошагового алгоритма работы контроллера в режиме поиска экстремальной мощности $[5,7]$. В режиме поиска экстремальной мощности контроллер считывает сигналы тока и напряжения СБ, а затем перемножает их. Если замер сигналов проводится в первый раз, то текущая мощность запоминается как мощность, полученная от СБ на предыдущем шаге.

Далее происходит увеличение коэффициента заполнения импульсов управления на некоторую фиксированную величину. После чего снова производится вычисление текущей мощности СБ. Если мощность увеличилась по сравнению с той, что запомнилась на предыдущем шаге, то происходит дальнейшее увеличение коэффициента заполнения. В случае, когда измеренная мощность уменьшается, изменяется направление движения рабочей точки по мощностной характеристике СБ, а следовательно, и уменьшается коэффициент заполнения импульсов управления.

Для проверки разработанных алгоритмов управления были изготовлены модель и макет контроллера СБ. Макет контроллера СБ состоит из силовой части, выполненной в виде конвертора понижающего типа $[5,8]$, и управляющего устройства, выполненного на основе микроконтроллера ATmega8, который формирует импульсы управления заданной длительности и частоты. Модель контроллера СБ реализована с помощью средств MatLAB/Simulink. Временные диаграммы токов и напряжений СБ, полученные при испытании модели и макета, показали полную работоспособность выведенных алгоритмов управления. 
Осциллограммы работы макета контроллера СБ в режиме поиска экстремальной мощности приведены на рис. 3. Напряжение СБ представлено на рисунке верхним графиком, ток СБ - нижним. Как видно из временных диаграмм, в момент времени 11 происходит включение режима поиска максимальной мощности, далее идет периодическое изменение коэффициента заполнения импульсов управления силовым ключом конвертора, и к моменту времени $\mathrm{t} 2$ максимальная мощность оказывается найденной, после чего осуществляются поисковые движения рабочей точки в окрестностях максимума мощности СБ (рис. 3б).

Временные диаграммы сигналов тока $I$ и напряжения $U$ солнечной батареи модели СЭП при переходе к режиму отбора экстремальной мощности даны на рис. 4. Представленные временные диаграммы сигналов тока $I$ и напряжения $U$ солнечной батареи модели СЭП аналогичны диаграммам, полученным при испытаниях макета СЭП, что подтверждает адекватность модели СЭП.

Абсолютная величина недоиспользования мощности $\Delta P_{c p}$ в режиме поиска экстремальной мощности рассчитывается по формуле

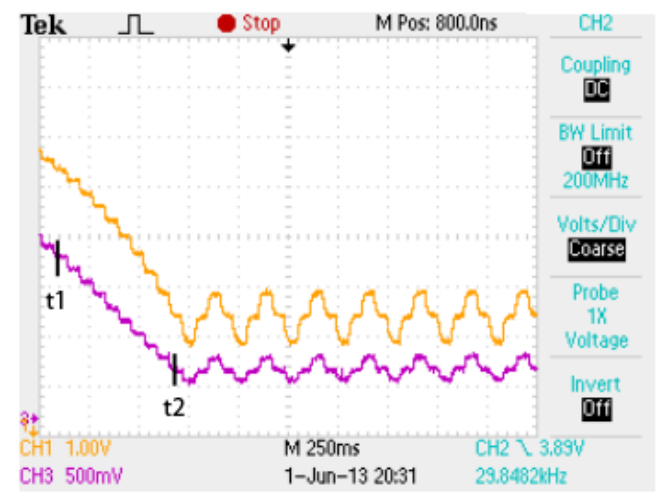

a

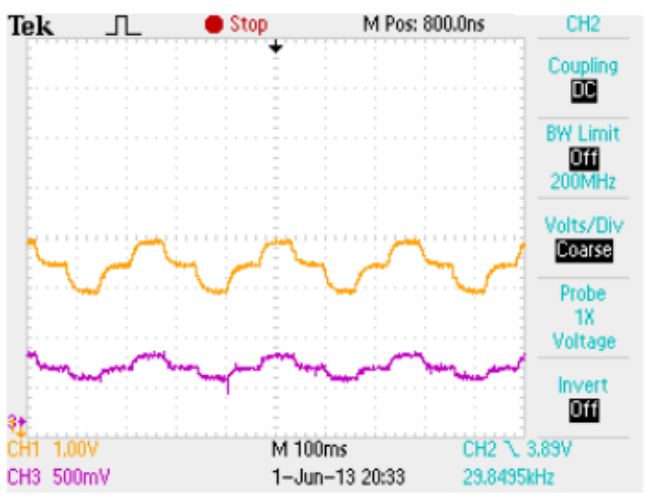

6

Рис. 3. Осциллограммы сигналов напряжения и тока СБ в режиме поиска максимальной мощности
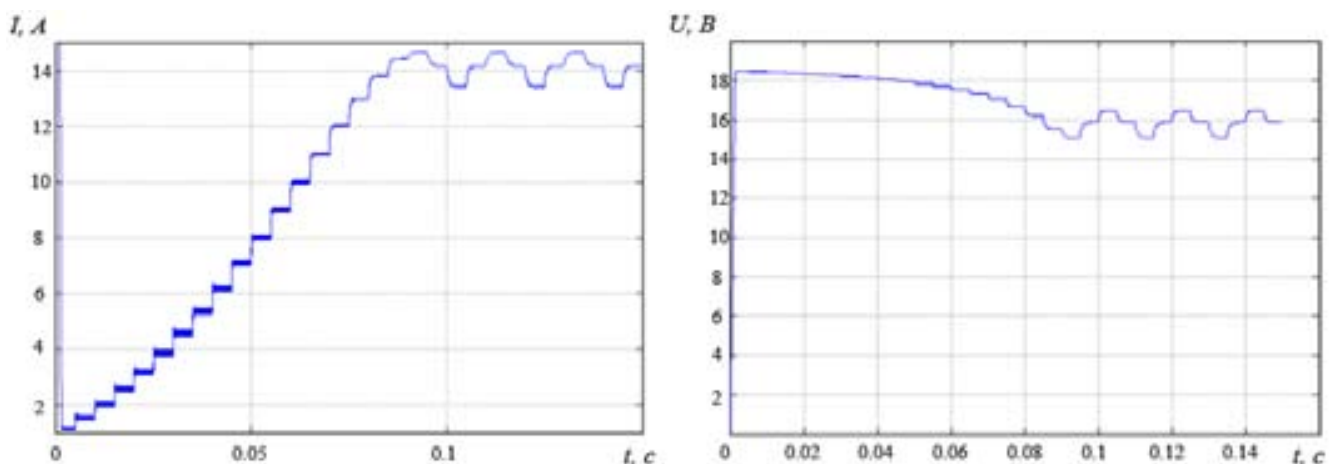

Рис. 4. Временные диаграммы сигналов тока $I$ и напряжения $U$ солнечной батареи модели СЭП в режиме поиска экстремальной мощности 


$$
\Delta P_{\mathrm{cp}}=\frac{1}{T} \int_{t_{1}}^{t_{2}}\left(P_{\max }-P(t)\right) d t,
$$

где $T$ - период колебаний рабочей точки; $t_{1}$ и $t_{2}-$ соответственно начало и конец одного периода колебаний рабочей точки; $P_{\max }$ - абсолютное значение максимума мощности СБ; $P(t)$ - текущая мощность СБ. Недоиспользование мощности СБ в результате работы алгоритма поиска экстремальной мощности составляет в среднем $1 \%$.

Достоинством вышеприведенного алгоритма поиска экстремальной мощности является то, что система управления осуществляет поиск и поддержание максимума мощности СБ практически при любых внешних условиях, независимо от вида и параметров ВАХ СБ. Основной недостаток в том, что колебания положения рабочей точки относительно максимума мощности СБ приводят также к некоторому недоиспользованию мощности СБ, величина которого возрастает по мере увеличения шага экстремального регулирования. Решить эту проблему можно, используя алгоритм поиска с переменным шагом. Ниже приведена математическая модель системы электропитания, алгоритм управления которой реализован с помощью средств теории нечетких множеств.

Математическая модель контроллера СБ построена с помощью средств программного пакета MATLAB/Simulink. Блок солнечных элементов Solar Cells состоит из нескольких последовательно включенных солнечных элементов [9]. Параметризация всех солнечных элементов выполняется по току короткого замыкания и напряжению холостого хода. Ток короткого замыкания одного элемента принят 7,34 А, а напряжение холостого хода - 0,8 В.

Алгоритм работы блока поиска экстремальной мощности (МРРТ) аналогичен приведенному на рис. 2 алгоритму работы экстремального регулятора с постоянным шагом. Дополнительным элементом в вышеприведенной СЭП служит непосредственно нечеткий контроллер, масштабирующий шаг экстремального регулятора в любом направлении поиска экстремальной мощности.

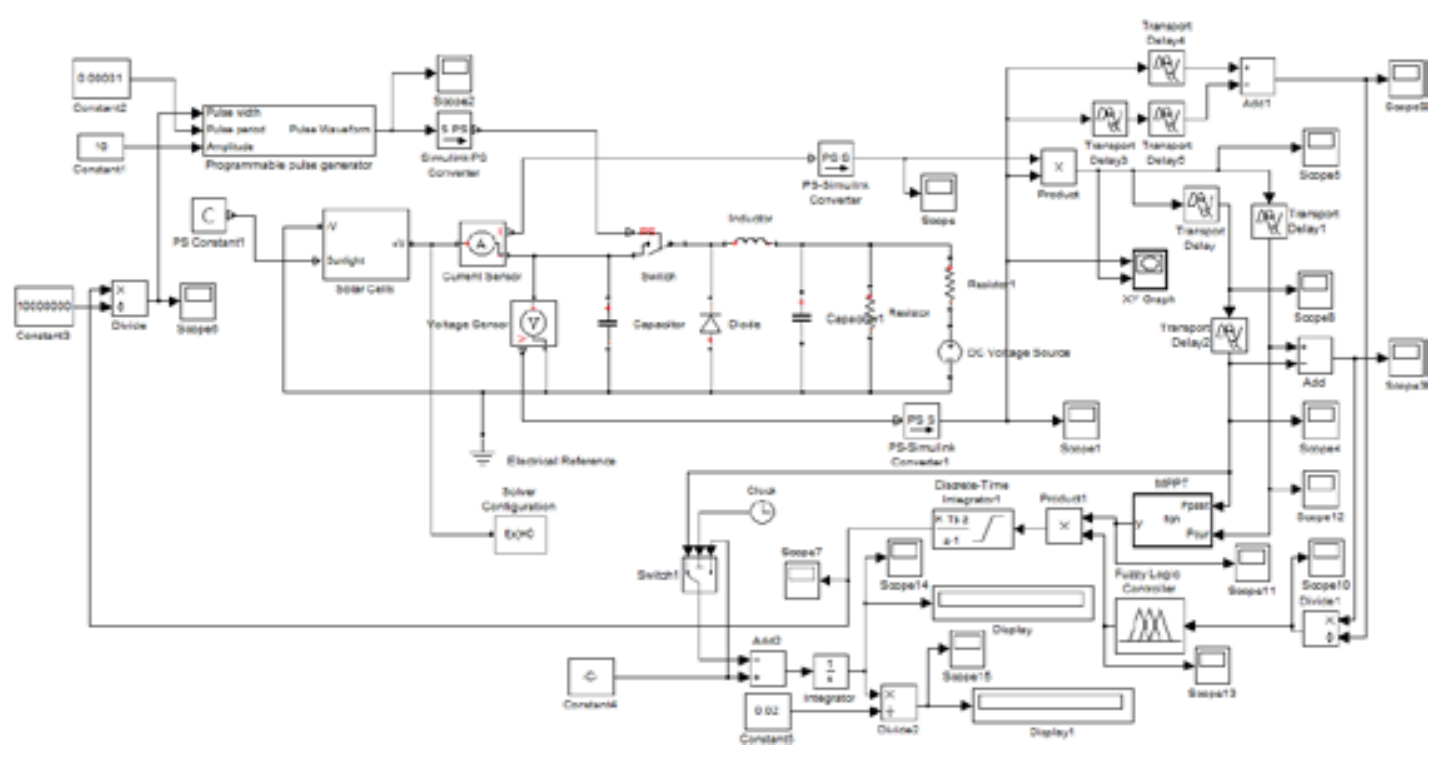

Рис. 5. Математическая модель контроллера СБ с нечетким регулятором

$$
-791-
$$


В качестве входной лингвистической переменной нечеткого контроллера принято отношение изменения мощности к изменению напряжения за один шаг экстремального регулятора, т.е. использован аналог производной мощности по напряжению $d P / d U$. Вид функций принадлежности входной и выходной переменной представлен на рис. 6 .

Выходная переменная нечеткого контроллера является коэффициентом, задающим величину шага ЭР (в данном случае лежащим в пределах от 0 до 12). Зависимость значения масштабирующего коэффициента от значения производной мощности по напряжению определяется набором правил нечеткого контроллера, который приведен в табл. 1.

Процесс перехода от функции принадлежности выходной переменной нечеткого контроллера к ее числовому значению, называемый также дефаззификацией, происходит с помощью метода центра тяжести [10]. Расчет числового значения выходной переменной нечеткого контроллера производится по формуле

$$
y=\frac{\int_{x_{\min }}^{x_{\max }} x \mu(x) d x}{\int_{x_{\min }}^{x_{\max }} \mu(x) d x},
$$

где $x$ - переменная, соответствующая выходной лингвистической переменной и принимающая значения от $x=x_{\min }$ до $x=x_{\max }$, при этом $x_{\min }$ и $x_{\max }$ соответственно левая и правая границы интервала значений нечеткого множества с ненулевой степенью принадлежности, $\mu(x)-$ функция принадлежности нечеткого множества.

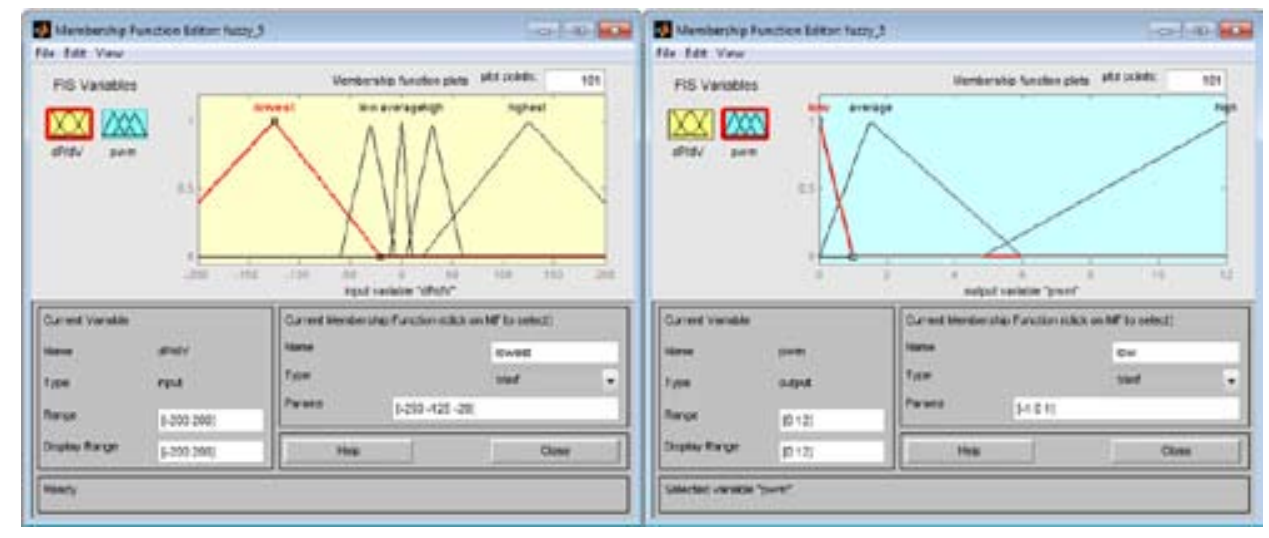

Рис. 6. Функции принадлежности входной и выходной переменной нечеткого контроллера

Таблица 1

\begin{tabular}{|c|c|}
\hline Значение $d P / d U$ & Значение выходной переменной \\
\hline Очень низкое & Высокое \\
\hline Низкое & Среднее \\
\hline Среднее & Низкое \\
\hline Высокое & Среднее \\
\hline Очень высокое & Высокое \\
\hline
\end{tabular}



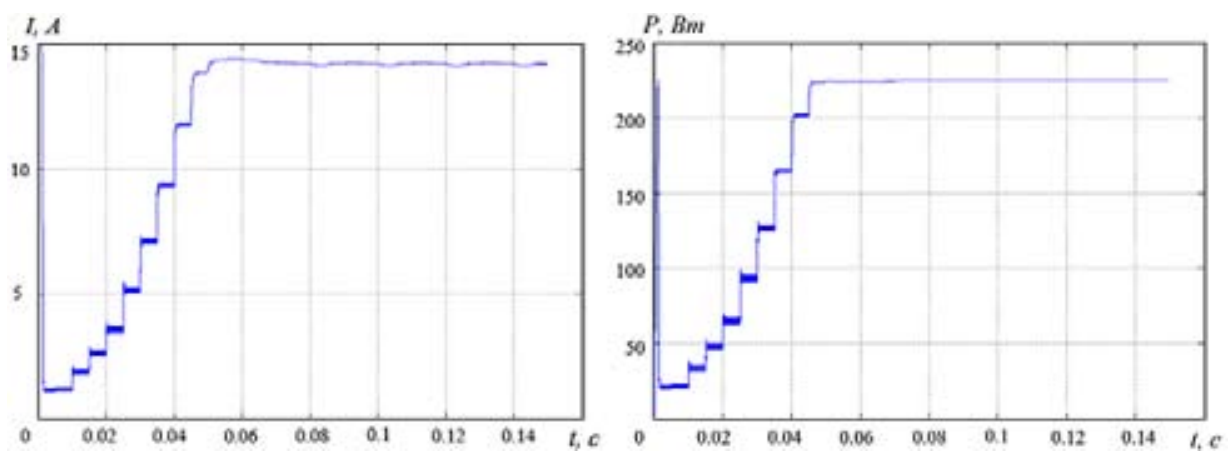

Рис. 7. Временные диаграммы изменения тока $I$ и мощности $P$ СБ в процессе работы алгоритма поиска экстремальной мощности нечеткого контроллера СБ

Использование переменного шага в работе алгоритма экстремального регулирования мощности позволяет увеличить скорость поиска экстремума и уменьшить амплитуду колебаний рабочей точки относительно точки максимума мощности. Временные диаграммы изменения тока $I$ и мощности Р СБ приведены на рис. 7.

Анализ временных диаграмм позволяет сделать вывод о том, что время, затраченное на поиск экстремума, существенно уменьшилось (с 90 до 45 мс), как и амплитуда колебаний рабочей точки относительно точки максимума. Недоиспользование мощности в режиме поиска экстремальной мощности с переменным шагом сократилось до $0,01 \%$. Параметры элементов СЭП, мощность нагрузки и освещенность СБ в модели с алгоритмом поиска с фиксированным шагом и модели с переменным шагом приняты одинаковыми.

\section{4. Заключение}

В ходе испытаний разработанного макета и модели контроллера СБ была подтверждена полная работоспособность алгоритма заряда АБ заданным током.

Сравнение эффективности работы контроллера СБ с алгоритмом поиска экстремума мощности СБ с фиксированным шагом и алгоритмом поиска экстремума с переменным шагом, проведённое с использованием имитационных моделей, показало снижение недоиспользования мощности солнечной батареи с 1 до $0,01 \%$ и снижение времени поиска экстремума на 50 \%. Такой результат достигается с помощью более совершенного алгоритма управления и не приводит к дополнительным затратам, связанным с совершенствованием силовой электрической схемы СЭП. Применение алгоритмов поиска экстремума с переменным шагом может быть актуальным как для мощных наземных систем электропитания, так и СЭП космических аппаратов.

\section{Список литературы}

[1] International Renewable Energy Agency: Vision and Mission. URL: http://www.irena.org/ menu/index.aspx?mnu=cat\&PriMenuID=13\&CatID=9 (дата обращения: 07.01.2015).

[2] Trends 2013 in photovoltaic applications. Survey report of selected IEA countries between 1992 and 2012. URL: http://helapco.gr/pdf/IEA_PVPS_Trends_Report_2013_v1_0_01.pdf(дата обращения: 10.01.2015).

$$
-793-
$$


[3] Carr G. Alternative energy will no longer be alternative. URL: http://www.economist.com/ news/21566414-alternative-energy-will-no-longer-be-alternative-sunny-uplands (дата обращения: 07.01.2015).

[4] Кенден К.В., Тремясов В.А. // Журнал СФУ. Техника и технологии. 2014 (7). № 8. $966-$ 975.

[5] Соустин Б.П., Иванчура В.И., Чернышев А.И., Исляев Ш.Н. Системы электропитания космических аппаратов. Новосибирск: Наука. Сибирская издательская фирма, 1994. 318 с.

[6] Пост С.С., Донцов О.А., Иванчура В.И., Краснобаев Ю.В. // Известия Томского политехнического университета. Техника и технология в энергетике. 2014. С. 114-120.

[7] Иванчура В.И., Краснобаев Ю.В., Донцов О.А., Пост С.С. // Электромеханические преобразователи энергии: Труды VI Междунар. науч.-техн. конф. ЭПЭ-2013. Томск, 2013. С. 180185.

[8] Донщов О.А. Использование экстремального регулятора в автономных системах электропитания. URL: http://conf.sfu-kras.ru/sites/mn2012/thesis/s006/s006-040.pdf (дата обращения: 26.12.2014).

[9] Ibbini M., Mansi S. // Computer Applications in Environmental Sciences and Renewable Energy. Kuala Lumpur, 2014. P. 97-103.

[10] Штовба С.Д. Введение в теорию нечетких множеств и нечеткую логику. URL: http:// matlab.exponenta.ru/fuzzylogic/book1/index.php (дата обращения: 01.05.2015). 